\title{
Design Scheme of Communication System to Monitor Equipments Working Condition
}

\author{
Yuping Xu, Jixue Xiao, Jianquan Gong, Qiang Zeng, Ze Wang, Chuike He, Zeng Jiang \\ School of Mechanical Engineering, Xihua University \\ Chengdu, China \\ 937376371@qq.com,xjx_163_com@163.com,617578567@qq.com, dayueyoutome@126.com, \\ 1136032119@qq.com,626199974@qq.com,1543926179@qq.com
}

\begin{abstract}
This paper puts forward a kind of communication system as a solution to monitor work condition of many process-control equipments, which can communicate in serial but have no their addresses, in comfortable office far from the equipments. As for the scheme, Modbus communication protocol and RS485, which have high reliability and strong anti-interference ability respectively, are employed and a communication controller accomplishes the networking of the equipments in an industrial site. The experimental results show that the scheme is practical and the communication system based on the scheme can perform the communication between the equipments in an industry site and the monitor severer running in PC that is far from the site.
\end{abstract}

Key words-monitor; communication in serial; working condition

\section{INTRODUCTION}

As household appliances and industrial machinery become more and more intelligent and networked, people's life and work become more and more easy and convenient. The accuracy of some old and heavy process-control equipments, which can communicate with other single device in serial but have no their addresses, may not be too high in comparison with the current similar device's. But the equipments can still accomplish heat treating or smelting, chemical process and so on with certain precision to meet the production requirements. Serving how to use well these machines for current production is attracting more and more attentions. Remote monitoring of the equipments can improve labor efficiency and save labor cost, as well as working environment and the quality of work ${ }^{[1]}$.

The machines should be identified so that they can be accessed. Generally, address is utilized to identify things in network. As for the machines without addresses, field bus can be employed in the networking. Every one of the equipments needs a bus node to configure the bus control, address the equipment, and complete the conversion between the field bus and serial bus, and convert related protocol, etc. A field bus controller is necessary to perform the communication between the he machines in a field site and monitor severer running in PC far from the site. The communication system is complex and not reliable, and the operation is inconvenient due to the nodes installation and

The Education Ministry of China; The Education Department of Sichuan Province, China; The Department of Sichuan Province Human Resources and Social Security, China; Xihua University, China maintenance ${ }^{[2-4]}$. Current serial bus technology and Microprocessor technology can also be utilized directly to implement the communication and this would encounter the similar questions. In fact, the processing capacity of the microprocessors in each node and communication controller is far from fully utilized. In order to make the best of the powerful capacity of microprocessor in communication controller and avoid above failings, we present a solution without nodes and with high reliability.

\section{BASIC STRUCTURE OF SYSTEM}

The communication system includes PC computer, interface converter, communication controller and several equipments have no their addresses. The system structure diagram is shown in figure 1. PC computer mainly provides the human-computer interaction interface, and controls the network access equipments, thus constructing a perfect network information management system manages the data. Interface converter is mainly used to shift electrical level between PC and communication controller. Communication controller is mainly to identify the devices and completes the communication protocol conversion between Modbus and the equipment protocol, so as to monitor work condition of the equipments in an industrial site. The communication controller mainly includes power circuit, single-chip microcomputer minimum system, watchdog circuit, key circuit, display circuit, storage circuit, the first level conversion circuit, the second level conversion circuit, address selection circuit, address identification circuit, etc.

To form a communication path between the PC and the communication controller, the first level circuit at one end connects to a serial port of single-chip microcomputer minimum system, at the other end is connected to a interface converter that connected to the PC. Single-chip microcomputer minimum system constructs communication path between controller and the equipments through the address selection circuit, and learns the equipments applying for communication through address identification circuit. The second level conversion circuit at one end connects to other serial port of single-chip microcomputer minimum system, at the rest of end are connected to a number of equipments through the corresponding RS232 serial port.

When the PC sends process parameters or instructions to the equipment, first of all, the information are sent to the communication controller in the form of the Modbus protocol $^{[5-6]}$. And then communication controller converts 
the Modbus protocol to the protocol can be the equipment to identify. Finally the information transformed are sent to the equipment. When one or more sets of equipments send the information to a PC such as working condition, first of all the information are sent to the communication controller. And then communication controller converts the protocol can be the equipments to identify to the Modbus protocol. Finally the information transformed is send to the PC. That is to say, the communication controller achieves the protocol conversion, information transmission, address selection from the devices, address identification from the devices sending information, so that the PC can communicate with the equipments.

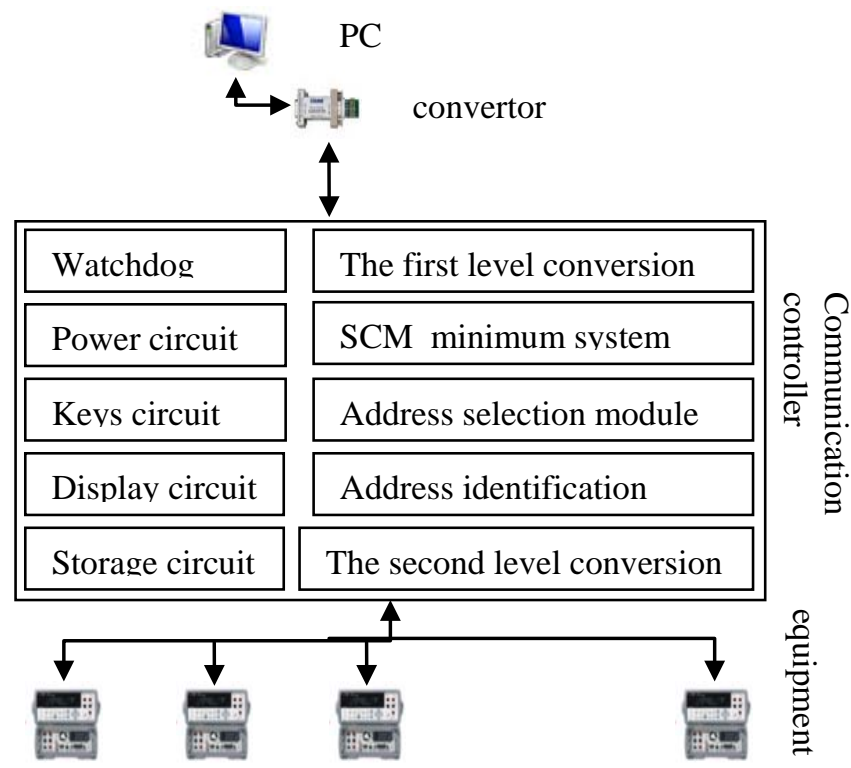

Fig 1 . The system structure diagram of communication system

\section{CIRCUIT MODULE}

\section{A. The first electrical level conversion circuit}

PC transfer information to communication controller by the transmission cable. In general, communication controller is installed in industrial field, the distance between PC and communication controller is longer, RS232 serial interface bus is suitable that the communication distance between the devices is less than $15 \mathrm{~m}$, transfer rate is less than $20 \mathrm{~KB} / \mathrm{s}$, and that often will be affected by the extra electrical interference and make mistakes ${ }^{[7]}$. So we choose RS485 serial interface to communicate, that has a strong antijamming ability. RS485 transmit signal by potential difference between the signal wires, the transmission distance is long, the speed is fast, transfer rate can reach 10 $\mathrm{MB} / \mathrm{s}$ biggest, maximum distance can reach $1200 \mathrm{~m}^{[8]}$.

Because of the single chip microcomputer using TTL level, when PC transmit information to MCU through RS232/RS485 interface converter, it is necessary that RS485 and TTL level can be transformed from and to each other. The circuit can realize RS485 and TTL level transformation is defined as the first level conversion circuit. This circuit is mainly composed of MAX485 chip and its affiliated circuit.
So using the RS232 / RS485 converter and the first level conversion circuit can realize a long information transmission between the PC and communication controller.

\section{B. Address selection circuit}

Address selection circuit of communication controller is used to complete the choice of the channel between the PC and equipments, so as to realize network communication between PC and the equipments under the control of the microcontroller. For example, when the PC communicates with the device 1, single chip microcomputer controls address selection circuit, that realizes the choice of information channel, so as to realize information transmission between the PC and the device 1 . The circuit is mainly composed of multiple ADG1406 chip and its affiliated circuit.

\section{Address identification circuit}

Address identification circuit of communication controller has such features: when a device sends information to the PC, recording address of sending information of the device, and that transmits information to the PC under the control of single-chip microcomputer according to certain rules. For example, when the device 1 and device 2 send information to the PC at the same time, MCU control address identification circuit to identify and record address and realizes the choice of information channel according to certain order, so as to realize information transmission between the PC and device 1 or 2 . This circuit is mainly composed of multiple 74HC32 chip, 8255A chip, and the auxiliary circuit.

\section{The second electrical level conversion circuit}

When the communication controller transmits information to the equipments, the distance between communication controller and equipment is extremely close, so RS232 interface can be directly used to realize asynchronous communication. In the same way, because of the microcontroller using TTL level and equipment using RS232 level, it is necessary that RS232 and TTL level can be transformed from and to each other, The circuit can realize RS232 and TTL level transformation is defined as the second level conversion circuit. The circuit is mainly made up of MAX232 chip and its affiliated circuit.

\section{CONCLUSION}

This paper introduces the basic structure and function of communication system, and the part of the circuit module of communication controller. This system makes full use of the processing capacity of the communication controller of single chip microcomputer, the address selection and identification circuit realize neatly recognition and communication between PC and serial equipments, the application of the Modbus protocol and RS485 bus possess very high anti-interference ability for network communication, especially so in industrial field.

Communication controller of single-chip microcomputer minimum system selects the STC12C5A60S2 single chip microcomputer as the core, MCU of control program and communication protocol are written in $\mathrm{C}$ language, and on 
the basis the laboratorial platform is built, and circuits are debugged. Hereafter, relevant experimental research is conducted, Take 5 equipments, for instance. The results show that this system can respond to information from 5 equipments in a timely manner and can realize reliably and precise transformation the equipment technological process and related parameters. The error of information transmission is not found and run good in the experiment.

\section{Acknowledgment}

This work is supported by the supported by the Key Scientific Research Project (grant number: Z2012015) from the Education Ministry of China, the Key Scientific Research Project(grant number: 13ZA0025) from the Education Department of Sichuan Province, China, and the Key Scientific Research Project (grant number: [2013] 203) from the Department of Sichuan Province Human Resources and Social Security, China, and Graduate Student Innovation Fund from Xihua University (grant number: YCJJ2015084), China.

\section{References}

[1] Wang dongsheng. Design of the Multi-protocol Communication Adapter Based on the Control Network. Inner Mongolia University of Science \&Technology,2015.

[2] Silva M, Pereira F, Soares F, Leão C.P, Machado J, Carvalho V. An overview of industrial communication networks. Mechanisms and Machine Science, v 24, p 933-940, 2015

[3] Zhang zhen, Niu yugang. Brief Introduction to DCS and the Fieldbus Technology. Electrical Automation,2013,01:4-6+46.

[4] Zeng Yidi, Schrom Harald, ErnstRolf . An approach for physical topology exploration in wired bus networks. Proceedings - IEEE International Symposium on Circuits and Systems, p 205-208, 2015

[5] Modbus Application Protocol V1 1b3. http://www.modbus.org

[6] Deng yuansheng. Based on single chip microcomputer of the modbus bus protocol implementation technology. Central South University,2009.

[7] Wu xiaoyan. Computer Serial Communication Interface and Its Application. Modern Electronics Technique, 2010, 83-86.

[8] Zhang Wenxiu, Lin Jun, Pen Lili, ChenJian. Application of RS485 for communication and synchronization in distributed electromagnetic exploration system. 2011 International Conference on Electric Information and Control Engineering, ICEICE 2011 Proceedings, p 4815-4818, 2011, 2011 International Conference on Electric Information and Control Engineering, ICEICE 2011 Proceedings 